\title{
The Distributed Detection Arithmetic with Fading Channel Based on The Minimum Error Probability
}

\author{
Yingkun Liu ${ }^{1}$ Xinxi Feng ${ }^{2}$ Honggang Dang ${ }^{3}$ Pingjun Pan ${ }^{4}$ \\ 1,2,4 The Telecommunication Engineering Institute, Air Force Engineering University, Xi'an 710077, P. R. China \\ ${ }^{3}$ The 94188th army, Xi'an 710077, P. R. China
}

\begin{abstract}
In the actual detection, due to bandwidth, channel fading and channel noise, the ideal channel isn't assured from the local detector to fusion center, so the optimal detection arithmetic is accordingly rectified. In this paper, we study the global optimal detection algorithm based on the two kinds of non-ideal channel schemes, firstly, channel state information is known; secondly, channel statistics characteristics is known. The configuration of the system is mixed with parallel and serial structure. The decision rules of the every local detector and every local processor must be optimized jointly based on the minimum error probability. At last the stimulation favors the analysis.
\end{abstract}

Keywords: Detection fusion, Non-ideal channel, Global optimization

\section{Introduction}

The study of distributed detection has traced back to 1960s, the effort significantly intensified since the publication of [1]. In [1], Tenney and Sandell formulated the distributed detection problem using a Bayesian setting. Later the distributed detection developed very quickly, This work was later generalized to multiple sensors by Reibman and Nolte [2] and by Hoballah、 Varshney [3]. Similarly, under the Neyman-Pearson (NP) criterion, the optimality of the local LRT has been established in [4]-[6]. In the above mentioned papers, all of them have an assumption: the local decisions are transmitted to fusion center with no error. Yet we must face to nonideal channel during the actual detection process owing to bandwidth 、 channel fading and channel noise, the non-ideal channel results in the error during the transmission, based on the error decision received in the fusion center, the performance using the above arithmetic[1]-[6] is degraded distinctly, so the optimal detection arithmetic is rectified accordingly. A few papers[7]-[9] have discussed the arithmetic involving of non-ideal channel in distributed detection, in [7][8] the optimal detection arithmetic of parallel and serial configuration have been studied based on binary symmetry channel, in [9] it is shown that the fusion center and local detectors are jointly optimized based on two non-ideal channel states in parallel configuration.

In order to enrich the system info of distributed detection, an optimized detection arithmetic is studied based on two kinds of non-ideal channel schemes in this paper, firstly, channel state information is known; secondly, channel statistics characteristics is known. In addition, the configuration of the system is the mixture of parallel and serial structure. According to the minimum error probability rule, the optimum decision rule of the local detectors and local processors are derived. At last, stimulation result shows that the performance of the second channel schemes suffer small performance loss compared with the first channel schemes, but its calculation is simplified and it is applied much more practically.

\section{System model}

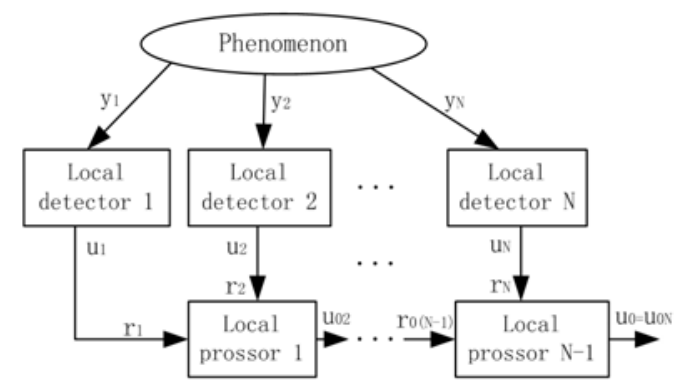

Fig.1: the system figure of the distributed detection.

Consider the problem of testing two hypotheses, denoted by $H_{0}$ (existing target) and $H_{1}$ (no target), with the prior probabilities $\pi_{0}$ and $\pi_{1}$ respectively. The distributed detection system comprises parallel $N$ local detectors 、 serial $N-1$ local processors and independent non-ideal channels, the system figure is shown in Fig.1, each local detector makes a decision $u_{i}(i=1,2 \ldots N)$ based on its own observation $y_{i}$, throughout this paper we assume that the observations 
$y_{i}(i=1,2 \ldots N)$ are conditionally independent, then this decision $u_{i}$ is transmitted to the corresponding local processor through a non-ideal channel, moreover the local processor makes a decision $u_{0 i}(i=2 \ldots N)$ based on the received decision $r_{0(i-1)}$ and $r_{i}$, then this decision $u_{0 i}$ is transmitted to the next local processor through a non-ideal channel, the output $u_{0 N}$ of local processor $N-1$ is the final decision $u_{0}$.

In this paper, the optimal decision rule of local detectors and local processors are derived based on minimized the average error probability defined as:

$P_{e 0}=\int_{g} \sum_{j=0}^{1} \pi_{j} P\left(u_{0}=1-j \mid H_{j}, g\right) p(g) d g$

$=\int_{g}\left(\pi_{0} P\left(u_{0}=1 \mid H_{0}, g\right)-\pi_{1} P\left(u_{0}=1 \mid H_{1}, g\right)+\pi_{1}\right) p(g) d g$

Where $g=\left\{g_{1}, \ldots g_{N}, g_{02}, \ldots g_{0(N-1)}\right\}$ is channel state information, $p(g)$ is probability density function of $g$. The remainder of the paper studies the optimized decision rule in following two kinds non-ideal channel schemes.

\section{The optimal decision rule based on channel state information}

We adopt a person-by-person optimization approach, namely we optimize the decision rule of a local detector or a local processor giving decision rules at all other local detectors and local processors fixed.

\subsection{The optimal decision rule of local processor}

In [10] the author testify that in serial topology configure if each local processor make an optimal decision then the system can obtain the optimal detection property. We can expand the average error probability in (1) with respect to the output $u_{0 i}$ and input $r_{0(i-1)} 、 r_{i}$ of local processor $i-1(2<i \leq N-1)$, and we get:

$$
\begin{aligned}
P_{e 0} & =\int_{g} \int_{r_{i}} \int_{r_{0(i-1)}}\left\{\left[\pi_{0} P\left(u_{0}=1 \mid u_{0 i}=0, H_{0}, g\right)\right.\right. \\
& p\left(u_{0 i}=0 \mid r_{i}, r_{0,(i-1)}\right) P\left(r_{i} \mid H_{0}\right) p\left(r_{0,(i-1)} \mid H_{0}\right)+ \\
& \pi_{0} P\left(u_{0}=1 \mid u_{0 i}=1, H_{0}, g\right) p\left(u_{0 i}=1 \mid r_{i}, r_{0,(i-1)}\right) \\
& \left.\left.P\left(r_{i} \mid H_{0}\right) p\left(r_{0,(i-1)} \mid H_{0}\right)\right]-P\left(r_{i} \mid H_{0}\right) p\left(r_{0,(i-1)} \mid H_{0}\right)\right]- \\
& {\left[\pi_{1} P\left(u_{0}=1 \mid u_{0 i}=0, H_{1}, g\right) p\left(u_{0 i}=0 \mid r_{i}, r_{0,(i-1)}\right)\right.} \\
& P\left(r_{i} \mid H_{1}\right) p\left(r_{0,(i-1)} \mid H_{1}\right)+\pi_{1} P\left(u_{0}=1 \mid u_{0 i}=1, H_{1}, g\right) \\
& \left.\left.p\left(u_{0 i}=1 \mid r_{i}, r_{0,(i-1)}\right) P\left(r_{i} \mid H_{1}\right) p\left(r_{0,(i-1)} \mid H_{1}\right)\right]+\pi_{1}\right\} \\
& p(g) d r_{i} d r_{0,(i-1)} d g
\end{aligned}
$$

Observing that $p\left(u_{0 i}=0 \mid r_{i}, r_{0,(i-1)}\right)=1-p\left(u_{0 i}=1 \mid r_{i}, r_{0,(i-1)}\right)$, substitute into (2) we have:

$$
\begin{aligned}
P_{e 0} & =\int_{g} C_{1}+\int_{r_{i}} \int_{r_{0(i-1)}}\left\{\pi_{0} A\left(u_{0}, u_{0 i}, H_{0}, g\right)\right. \\
& P\left(u_{0 i}=1 \mid r_{i}, r_{0,(i-1)}\right) P\left(r_{i} \mid H_{0}\right) p\left(r_{0,(i-1)} \mid H_{0}\right)- \\
& \pi_{1} A\left(u_{0}, u_{0 i}, H_{1}, g\right) P\left(u_{0 i}=1 \mid r_{i}, r_{0,(i-1)}\right) P\left(r_{i} \mid H_{1}\right) \\
& \left.p\left(r_{0,(i-1)} \mid H_{1}\right)\right\} p(g) d r_{i} d r_{0,(i-1)} d g
\end{aligned}
$$

where:

$$
\begin{aligned}
A\left(u_{0 N}, u_{0 i}, H_{1}, g\right)= & P\left(u_{0 N}=1 \mid u_{0 i}=1, H_{1}, g\right) \\
& -P\left(u_{0 N}=1 \mid u_{0 i}=0, H_{1}, g\right)
\end{aligned}
$$

According to [6], we obtain:

$\left\{\begin{array}{l}A\left(u_{0 N}, u_{0 i}, H_{j}, g\right)=\prod_{k=i+1}^{N} A\left(u_{0 k}, u_{0(k-1)}, H_{j}, g\right) \\ A\left(u_{0 N}, u_{0 i}, H_{j}, g\right)>0, \quad k=1,2, \ldots N-1, j=0,1\end{array}\right.$

Otherwise $C_{1}$ in (3)can be expressed:

$C_{1}=\pi_{0} P\left(u_{0}=1 \mid u_{0 i}=0, H_{0}, g\right)-\pi_{1} P\left(u_{0}=1 \mid u_{0 i}=0, H_{1}, g\right)+\pi_{1}$

obviously $C_{1}$ is a constant with regard to the decision rule of local processor $i-1(2<i \leq N-1)$.

To minimize $P_{e 0}$, from (3) the optimal decision rule for local processor $i-1(2<i \leq N-1)$ is:

$\frac{P\left(r_{i} \mid H_{1}\right) p\left(r_{0, i-1)} \mid H_{1}\right)}{P\left(r_{i} \mid H_{0}\right) p\left(r_{0,(i-1)} \mid H_{0}\right)} \frac{H_{H_{0}}}{<} \frac{\pi_{0} A\left(u_{0}, u_{0 i}, H_{0}, g\right)}{\pi_{1} A\left(u_{0}, u_{0 i}, H_{1}, g\right)}$

where

$\left\{\begin{array}{c}p\left(r_{i} \mid H_{j}\right)=\int_{g_{i}}\left[\sum_{u_{i}} p\left(r_{i} \mid u_{i}, g_{i}\right) P\left(u_{i} \mid H_{j}\right)\right] p\left(g_{i}\right) d g_{i} \\ p\left(r_{0,(i-1)} \mid H_{j}\right)=\int_{g_{(i-1)}}\left[\sum_{u_{0(i-1)}} p\left(r_{0,(i-1)} \mid u_{0,(i-1)}, g_{(i-1)}\right)\right. \\ \left.P\left(u_{0,(i-1)} \mid H_{j}\right)\right] p\left(g_{(i-1)}\right) d g_{(i-1)}\end{array}\right.$

Since there is no local processor before local processor 1 , the input of local processor 1 are the received decision of local detector 1 and 2, similarly, we can get the optimal decision rule for local processor 1 ,

$\frac{P\left(r_{1} \mid H_{1}\right) p\left(r_{2} \mid H_{1}\right)}{P\left(r_{1} \mid H_{0}\right) p\left(r_{2} \mid H_{0}\right)} \frac{H_{1}}{\underset{H_{0}}{<}} \frac{\pi_{0} A\left(u_{0}, u_{02}, H_{0}, g\right)}{\pi_{1} A\left(u_{0}, u_{02}, H_{1}, g\right)}$

\subsection{The optimal decision rule of local processor}

While we design the optimal decision rule of one local processor, at first we assume that all of the local processors and the other local detectors fix the decision rules.

According to probability theory, we have:

$P\left(r_{i} \mid H_{j}\right)=\int_{g} \int_{y_{1}} \sum_{u_{i}} P\left(r_{i} \mid u_{i}, g\right) P\left(u_{i} \mid y_{i}\right) p\left(y_{i} \mid H_{j}\right) p(g) d y_{i} d g, j=0,1$

Substituting (8)into (3),

$$
\begin{aligned}
P_{e 0}= & \int_{g} C_{1}+\int_{r_{i}} \int_{r_{0(i-1)}} \int_{y_{i}} \sum_{u_{i}}\left\{\pi_{0} A\left(u_{0}, u_{0 i}, H_{0}, g\right)\right. \\
& P\left(u_{0 i}=1 \mid r_{i}, r_{0,(i-1)}\right) p\left(r_{i} \mid u_{i}, g\right) P\left(u_{i} \mid y_{i}\right) p\left(y_{i} \mid H_{0}\right) \\
& p\left(r_{0,(i-1)} \mid H_{0}\right)-\pi_{1} A\left(u_{0}, u_{0 i}, H_{1}, g\right) P\left(u_{0 i}=1 \mid r_{i}, r_{0,(i-1)}\right) \\
& \left.p\left(r_{i} \mid u_{i}, g\right) P\left(u_{i} \mid y_{i}\right) p\left(y_{i} \mid H_{1}\right) p\left(r_{0,(i-1)} \mid H_{1}\right)\right\} \\
& p(g) d r_{i} d r_{0,(i-1)} d y_{i} d g
\end{aligned}
$$

obviously $C_{1}$ is a constant for local detector $i(i>2)$. To minimize $P_{e 0}$, one can see from (9) that the optimal decision rule for local detector $i(i>2)$ is:

$$
\frac{p\left(y_{i} \mid H_{1}\right)}{p\left(y_{i} \mid H_{0}\right)} \frac{{ }_{1}}{\geq} \frac{\pi_{0}}{<} \frac{\pi_{0}}{\pi_{1} A\left(u_{0}, u_{0 i}, H_{0}, g\right) p\left(r_{0,(i-1)} \mid H_{0}\right)}
$$

Due to the decisions of local detector 1、2 through non-ideal channel is the input of local processor 1 , 
similarly the optimal decision rule for local detector

1、 2 are:

$$
\begin{aligned}
& \frac{P\left(y_{1} \mid H_{1}\right)}{P\left(y_{1} \mid H_{0}\right)} \frac{u_{1}=1}{\underset{u_{1}=0}{<}} \frac{\pi_{0} A\left(u_{0 N}, u_{02}, H_{0}, g\right) P\left(r_{2} \mid H_{0}\right)}{\pi_{1} A\left(u_{0 N}, u_{02}, H_{1}, g\right) P\left(r_{2} \mid H_{1}\right)} \\
& \frac{P\left(y_{2} \mid H_{1}\right)}{P\left(y_{2} \mid H_{0}\right)} \frac{u_{2}=1}{\underset{u_{2}=0}{<}} \frac{\pi_{0} A\left(u_{0 N}, u_{02}, H_{0}, g\right) P\left(r_{1} \mid H_{0}\right)}{\pi_{1} A\left(u_{0 N}, u_{02}, H_{1}, g\right) P\left(r_{1} \mid H_{1}\right)}
\end{aligned}
$$

From (10)-(12), we can see that the likelihood ratio test threshold of local detector $i(i>2) 、 1 、 2$ has relation to $r_{0(i-1)} 、 r_{2} 、 r_{1}$ respectively.

\section{The optimal decision rule on channel statistical property}

Although the optimal decision rule for each sensor is explicitly formulated in (5)(7)(10)(11)(12), the likelihood ratio thresholds are intractable, as they involve highly nonlinear function g, The only possible way of finding the optimal decision rules of local processors and detectors appears to be an exhaustive search, whose complexity becomes prohibitive when $N$ is large.

Instead of directly minimizing the average error probability as in (3), an alternative approach is to first average the channel transition probability with respect to the fading channel. That is, we compute $p\left(r_{i} \mid u_{i}\right),(i=1, \ldots N) \quad$ and $\quad p\left(r_{0 i} \mid u_{0 i}\right),(i=2, \ldots N) \quad$ by marginalizing out the channel density function $p\left(g_{i}\right)$,namely,

$p\left(r_{i} \mid u_{i}\right)=\int_{g_{i}} p\left(r_{i} \mid u_{i}, g_{i}\right) p\left(g_{i}\right) d g_{i}, \quad i=1, \ldots N$

$p\left(r_{0 i} \mid u_{0 i}\right)=\int_{g_{i}} p\left(r_{0 i} \mid u_{0 i}, g_{(i)}\right) p\left(g_{(i)}\right) d g_{(i)}, i=2, \ldots N-1$

According to (13)(14), we can obtain the

$P\left(u_{0}=1 \mid u_{0 i}=j, H_{k}\right),(j, k=0,1 ; i=2, \ldots N-1)$

by marginalizing out the channel $g$ :

$P\left(u_{0}=1 \mid u_{0 i}=j, H_{k}, g\right)=\int_{g} P\left(u_{0}=1 \mid u_{0 i}=j, H_{k}, g\right) p(g) d g$

Therefore we gain $A\left(u_{0}, u_{0 i}, H_{k}\right)$.Similarly we have a result resembling that of knowing the channel state scheme, the optimal decision rules of local processor $i-1(2<i \leq N-1)$ 、 local processor 1 、 local detector $i(i>2)$ 、 local detector 2 、 local detector 1 are respectively:

$$
\begin{aligned}
& \frac{P\left(r_{i} \mid H_{1}\right) p\left(r_{0,(i-1)} \mid H_{1}\right)}{P\left(r_{i} \mid H_{0}\right) p\left(r_{0,(i-1)} \mid H_{0}\right)} \underset{\substack{u_{0 i}=1 \\
u_{0 i}=1}}{>} \frac{\pi_{0} A\left(u_{0}, u_{0 i}, H_{0}\right)}{\pi_{1} A\left(u_{0}, u_{0 i}, H_{1}\right)} \\
& \frac{P\left(r_{1} \mid H_{1}\right) p\left(r_{2} \mid H_{1}\right)}{P\left(r_{1} \mid H_{0}\right) p\left(r_{2} \mid H_{0}\right)} \frac{\stackrel{u_{02}=1}{>} \underset{u_{02}=0}{<}}{\pi_{0} A\left(u_{0}, u_{02}, H_{0}\right)} \\
& \frac{p\left(y_{i} \mid H_{1}\right)}{p\left(y_{i} \mid H_{0}\right)} \frac{\stackrel{u_{i}=1}{>}}{\underset{u_{i}=0}{<}} \frac{\pi_{0} A\left(u_{0}, u_{0,(i-1)}, H_{0}\right) p\left(r_{0,(i-1)} \mid H_{0}\right)}{\pi_{1} A\left(u_{0}, u_{0,(i-1)}, H_{1}\right) p\left(r_{0,(i-1)} \mid H_{1}\right)}
\end{aligned}
$$

$$
\begin{aligned}
& \frac{P\left(y_{1} \mid H_{1}\right)}{P\left(y_{1} \mid H_{0}\right)} \frac{\stackrel{u_{1}=1}{>}}{\underset{u_{1}=0}{<}} \frac{\pi_{0} A\left(u_{0 N}, u_{02}, H_{0}\right) P\left(r_{2} \mid H_{0}\right)}{\pi_{1} A\left(u_{0 N}, u_{02}, H_{1}\right) P\left(r_{2} \mid H_{1}\right)} \\
& \frac{P\left(y_{2} \mid H_{1}\right)}{P\left(y_{2} \mid H_{0}\right)} \frac{{ }_{u_{2}=1}^{>}}{\underset{u_{2}=0}{<}} \frac{\pi_{0} A\left(u_{0 N}, u_{02}, H_{0}\right) P\left(r_{1} \mid H_{0}\right)}{\pi_{1} A\left(u_{0 N}, u_{02}, H_{1}\right) P\left(r_{1} \mid H_{1}\right)}
\end{aligned}
$$

\section{Simulation}

In this section, a two-local-processor and three-localdetector example is uesed to evaluate the detection property with two kinds channel schemes. Consider the detection of a known signal $s$ in zero-mean Gaussian noises that are independent and identically distributed (i.i.d.) for the three local detectors.

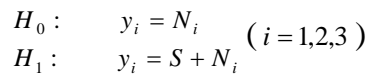

Where $N_{1}, N_{2}, N_{3} \sim C N\left(0, \sigma_{1}^{2}\right)$, Without loss of generality, we assume $S=1, \sigma_{1}^{2}=2$.

Each sensor makes a binary decision $u_{i}$ based on its observation $y_{i}$, and then transmits it through a Rayleigh fading channel to the corresponding local processor. The channel output is

$r_{i}=g_{i} u_{i}+W_{i}$

where $g_{1}, g_{2}, g_{3}$ are i.i.d. and zero-mean Gaussian distributed $g_{1}, g_{2}, g_{3} \sim C N\left(0, \sigma_{g}^{2}\right), \sigma_{g}^{2}=1, W_{1}, W_{2}, W_{3}$ are i.i.d. and zero-mean Gaussian noises with distribution $W_{1}, W_{2}, W_{3} \sim C N\left(0, \sigma_{w}^{2}\right), \sigma_{W}^{2}=1$.

In addition, the decision of local processor 1 is transmitted to local processor 2 through a Rayleigh fading channel, the channel output is:

$r_{02}=g_{02} u_{02}+W_{02}$

where $g_{02}, W_{02} \sim C N\left(0, \sigma_{02}^{2}\right)$ are i.i.d. we assume $\sigma_{02}^{2}=1$.

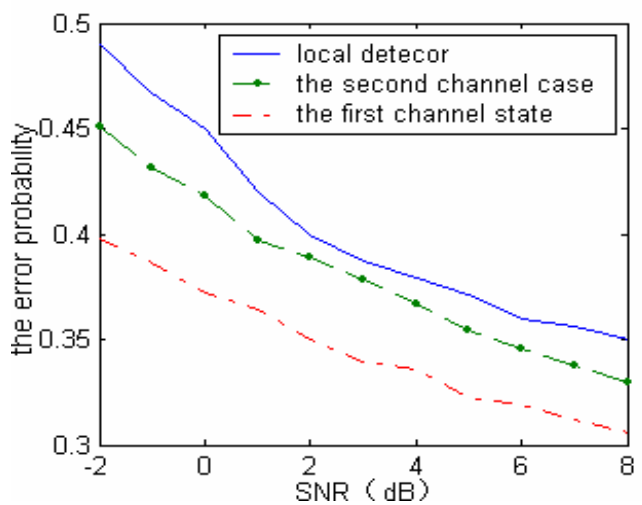

Fig.2: the error probability versus SNR.

If prior probabilities $\pi_{1}=\pi_{0}$, the average error probabilities is a function of the average signal-tonoise ratio (SNR) of the received signal, Fig.2 shows that the error probability of local detectors and local 
processors are affected by the SNR. From Fig.2, we can see the property of local processor is better than local detector, the property of knowing channel state information is better than that of knowing channel statistical performance, but the calculation of the second case becomes simple, this is very important especially to large $N$.

\section{Conclusions}

In this paper, the topology configuration is mixture of parallel and serial structure, in two channel cases, we derive the optimal decision rule of all sensors based on minimized the globe error probability. By stimulation we can know that though the second channel scheme suffer small performance loss compared with the first scheme, it overcome the problem of calculation intricacy, to more important, the second case has more practicability: firstly, the exact instant channel state can not be obtained accurately, however the statistical property of channel can gained by quantified sampling approximately; secondly if the channel state changes, but the channel statistical property remains unchanged, the optimal decision rule of the second channel case keeps applicable.

\section{References}

[1] R.R.Tenney, N.R.Sandell,Jr., Detection with distributed sensors, IEEE Trans. on AES, 17(2):501-510,1981.

[2] A.R Reibman,and L.W.Nolte, Optimal detection and performance of distributed sensor systems, IEEE Trans. on AES, 23(1):24-30,1987.

[3] I.Y. Hoballah and P.K.Varshney, Distrbuted Bayesian signal detection, IEEE Trans. Inform. Theory ,35(9):995-1000,1989.

[4] M. Xiang, J.W. Zhao, On the performance of distributed Neyman-Pearson detection systems. IEEE Trans. on Systems, Man, and Cybernetics. 31(1):78-83,2005.

[5] X.G. liang, Research on global optimization arithmetic for distributed detection system, Journal of bomb and Rocket \& control and guide,26(3):182-185,2006.

[6] M. Xiang, On optimum distributed detection and robustness of system performance, ISIF:156163,2002.

[7] B. Chen, P. K.Willett, On the optimality of the likelihood -ratio test for local sensor decision rules in the presence of non-ideal channels, IEEE Trans Inform Theory, IT - 51:693 - 699, 2005.

[8] X.G. Liang, Research on fusion algorithm for a kind of two-sensor distributed detection system with non - ideal communication channel, Journal of Detection \& Control, 28(4):30-33, 2006.

[9] B.Liu, Decentralized detection in wireless sensor networks with channel fading statistics, ICASSP: IV 857-IV 860, 2006.

[10]R. Viswanathan, Optimal serial distributed decision fusion, IEEE Trans on AES, 24(4):366375, 1988. 